\title{
Cohort profile: The Hoveyzeh Cohort Study (HCS): A prospective population-based study on non-communicable diseases in an Arab community of Southwest Iran
}

\author{
Bahman Cheraghian ${ }^{1}$, Seyed Jalal Hashemi ${ }^{2}$, Seyed Ahmad Hosseini ${ }^{3}$, Hossein Poustchi ${ }^{4}$, Zahra Rahimi ${ }^{5}$, \\ Sara Sarvandian ${ }^{5}$, Amal Saki Malehi ${ }^{5}$, Meysam Alipour $^{6}$, Sareh Eghtesad ${ }^{4}$, Jafar Fatahiasl ${ }^{7}$, Arash Bayat $^{8}$, \\ Hanieh Raji ${ }^{9}$, Nader Saki*10 (1)
}

Received: 15 Aug 2020

Published: 21 Oct 2020

\begin{abstract}
Background: The Hoveyzeh cohort study (HCS) is a population-based cohort study that conducted in Hoveyzeh County (South-west Iran). HCS focus on common chronic diseases, disorders and risk factors of NCDs in the Arab ethnicity.

Methods: A total number of 10009 participants (35-70 years old) were recruited in this prospective cohort study from May 2016 to August 2018. The HCS data were gathered by trained interviewer through interviewer-administered questionnaires. Also anthropometric measurements, physical examinations, clinical assessments, ophthalmology evaluation, auditory examinations, respiratory and cardiovascular assessments was conducted by means of standard instruments. Biological samples including blood, urine, hair, and nail collected and stored in the biobank.

Results: The overall participation rate was $82.7 \%$. The prevalence of obesity was $27.4 \%$ in males and $47 \%$ in females. Cigarette smoking prevalence was $20.9 \%$ ( $40.6 \%$ in men and $7.6 \%$ in women). Prevalence of major non communicable diseases such as diabetes, hypertension, metabolic syndrome, cardiac ischemic, myocardial infarction and stroke was $22.2 \%, 26.4 \% 31.9 \%, 13.6 \%, 1.85 \%$ and $1.6 \%$ respectively.

Conclusion: Considering the high prevalence of obesity and smoking in the population of Hoveyzeh and since the important role of these risk factors in development of common non communicable diseases, this issue should be taken into consideration and the necessary interventions in this context must be considered to modify lifestyle. The HCS is the only comprehensive cohort in the region, enabling it to provide valuable evidence about NCDs for a wide geographical area covering millions of people in both Iran and Iraq.
\end{abstract}

Keywords: The Hoveyzeh cohort study, HSC, Non-communicable diseases, Cohort profile, Prospective population-based study, Southwestern Iran, PERSIAN

Conflicts of Interest: None declared

Funding: The Iranian Ministry of Health and Medical Education has contributed to the funding used in the PERSIAN Cohort through Grant no. 700/534. Ahvaz Jundishapur University of Medical Sciences has also substantially contributed to the funding.

\section{*This work has been published under CC BY-NC-SA 1.0 license.}

Copyright $\odot$ Iran University of Medical Sciences

Cite this article as: Cheraghian B, Hashemi SJ, Hosseini SA, Poustchi H, Rahimi Z, Sarvandian S, Saki Malehi A, Alipour M, Eghtesad S, Fatahiasl J, Bayat A, Raji H, Saki N. Cohort profile: The Hoveyzeh Cohort Study (HCS): A prospective population-based study on non-communicable diseases in an Arab community of Southwest Iran. Med J Islam Repub Iran. 2020 (21 Oct);34:141. https://doi.org/10.47176/mjiri.34.141

\section{Corresponding author: Dr Nader Saki, saki-n@ajums.ac.ir}

1. Alimentary Tract Research Center, Department of Biostatistics \& Epidemiology, School of Public Health, Ahvaz Jundishapur University of Medical Sciences, Ahvaz, Iran

2. Alimentary Tract Research Center, School of Medicine, Ahvaz Jundishapur University of Medical Sciences, Ahvaz, Iran

3. Nutrition and Metabolic Diseases Research Center, Department of Nutrition, School of Allied Medical Sciences, Ahvaz Jundishapur University of Medical Sciences, Ahvaz, Iran

4. Liver and Pancreatobiliary Diseases Research Center, Digestive Diseases Research Institute, Tehran University of Medical Sciences, Tehran, Iran

5. Department of Biostatistics \& Epidemiology, School of Public Health, Ahvaz Jundishapur University of Medical Sciences, Ahvaz, Iran

6. Nutrition and Metabolic Diseases Research Center, Ahvaz Jundishapur University of Medical Sciences, Ahvaz, Iran

7. Department of Radiology Technology, School of Allied Medical Sciences, Ahvaz Jundishapur University of Medical Sciences, Ahvaz, Iran

8. Hearing Research Center, Ahvaz Jundishapur University of Medical Sciences, Ahvaz, Iran

9. Department of Internal Medicine, Air Pollution and Respiratory Diseases Research Center, Ahvaz Jundishapur University of Medical Sciences, Ahvaz, Iran

10. Department of Otolaryngology, Head and Neck Surgery, Hearing Research Center, Ahvaz Jundishapur University of Medical Sciences, Ahvaz, Iran $\uparrow$ What is "already known” in this topic:

Although, the STEPS program about NCDs risk factors has already been performed on a small sample size of the Hoveyzeh population, but no comprehensive health screening program has been conducted in this area so far.

$\rightarrow$ What this article adds:

This study is the first and only comprehensive health research on total adult's residents (35-70 years old) in this county. The prevalence of common NCDs like Hypertension, Diabetes and Metabolic Syndrome were identified. Also, the prevalence of major risk factors for NCDs such as obesity cigarette smoking were determined. 


\section{Why was Hoveyzeh cohort set up?}

Non-communicable diseases (NCDs) cause 41 million annual deaths worldwide (1). NCDs account for $72.3 \%$ of total deaths in 2016 (2). It is estimated that $82 \%$ of all deaths in Iran are caused by NCDs, including cardiovascular diseases $(43 \%)$, cancers (16\%), chronic respiratory diseases $(4 \%)$, diabetes $(4 \%)$, accidents $(10 \%)$, and other NCDs (15\%) (3). In 2017, low back pain, headache, and depression were reported as 3 major causes of YLD (Years Lived with Disability) (4). In developed and developing countries, NCDs impose a heavy burden on health systems and the economy of communities. It is estimated that NCDs will cost more than $\$ 30$ trillion in the next 2 decades, which is equivalent to $48 \%$ of GDP in 2010 pushing millions into poverty (5). The transition from infectious diseases to NCDs in developing countries is the outcome of some risk factors often caused by economic progress. The 4 major risk factors in 2017 were hypertension, smoking, high blood sugar, and high body mass index (BMI) (2). Tobacco use, insufficient physical activity, alcohol abuse, and inadequate nutrition increase the risk of death from NCDs (1, 6). Numerous cohort studies have been conducted on large populations to explore the association between non-communicable disease risk factors in developed countries, but such studies have been implemented only in a few developing countries. No population-based cohort study has been conducted to date on non-communicable diseases in the southwest region of Iran (Khuzestan) and in the Arab ethnicity. Moreover, the mechanisms of disease development may vary based on geographical, cultural, and social conditions, so this comprehensive and prospective study seems necessary in the region. The Hoveyzeh cohort study (HCS) will focus on common chronic diseases or disorders, such as cardiovascular, respiratory, digestive, hepatic, renal, metabolic, psychiatrics, visual, hearing and cancers, in adults. Moreover, it will assess the effects of demographic, socioeconomic, genetic, nutritional, and environmental and lifestyle factors of the common chronic diseases or disorders as well as their interactions. The objectives of our study are to estimate the prevalence and incidence of NCDs, to determine crude death rate and cause-specific mortality rates for the main causes of mortality, to define the natural history of NCDs accurately, to discover the risk and protective factors of NCDs in the Arab community, to establish a population-based research context for health researchers, and to provide a Biobank for assessing the role of biomarkers and genes in disease and death (7).

Khuzestan province is located in Southwest Iran, bordering Iraq in the west and the Persian Gulf in the south. Hoveyzeh county is located on the west side of Khuzestan province with a total area of $5330 \mathrm{~km}^{2}$ (Fig. 1). The county is located about 80 kilometers from Ahvaz, the capital of Khuzestan. The climate is generally very hot and occasionally humid so that the temperature varies from $55^{\circ} \mathrm{C}$ in the summer to $10^{\circ} \mathrm{C}$ in the cold seasons. Most of the residents are from the Arab ethnicity. The county is divided into 2 districts: the Central District and Neysan District. The largest wetland in Khuzestan, called Houralazim or Houralhoveyzeh, is located in this area. Agricultural and pastoral occupations are very common jobs in the county. Hoveyzeh suffered very heavy damages during the Iran-Iraq war, resulting in the complete destruction of the city. Due to environmental changes such as drought, the wetland has become a source of dust; also, climatic changes led to wildfires in the wetland that might be one of the major risk factors threatening the health of its inhabitants, especially the incidence of respiratory diseases. Moreover, the presence of numerous oil and gas fields may cause health-related ecological changes in this region. Moreover, there are many sunshine days in Hoveyzeh each year, so high levels of exposure to sunlight can increase the risk of skin and eye diseases, especially among outdoor workers. The grant of this

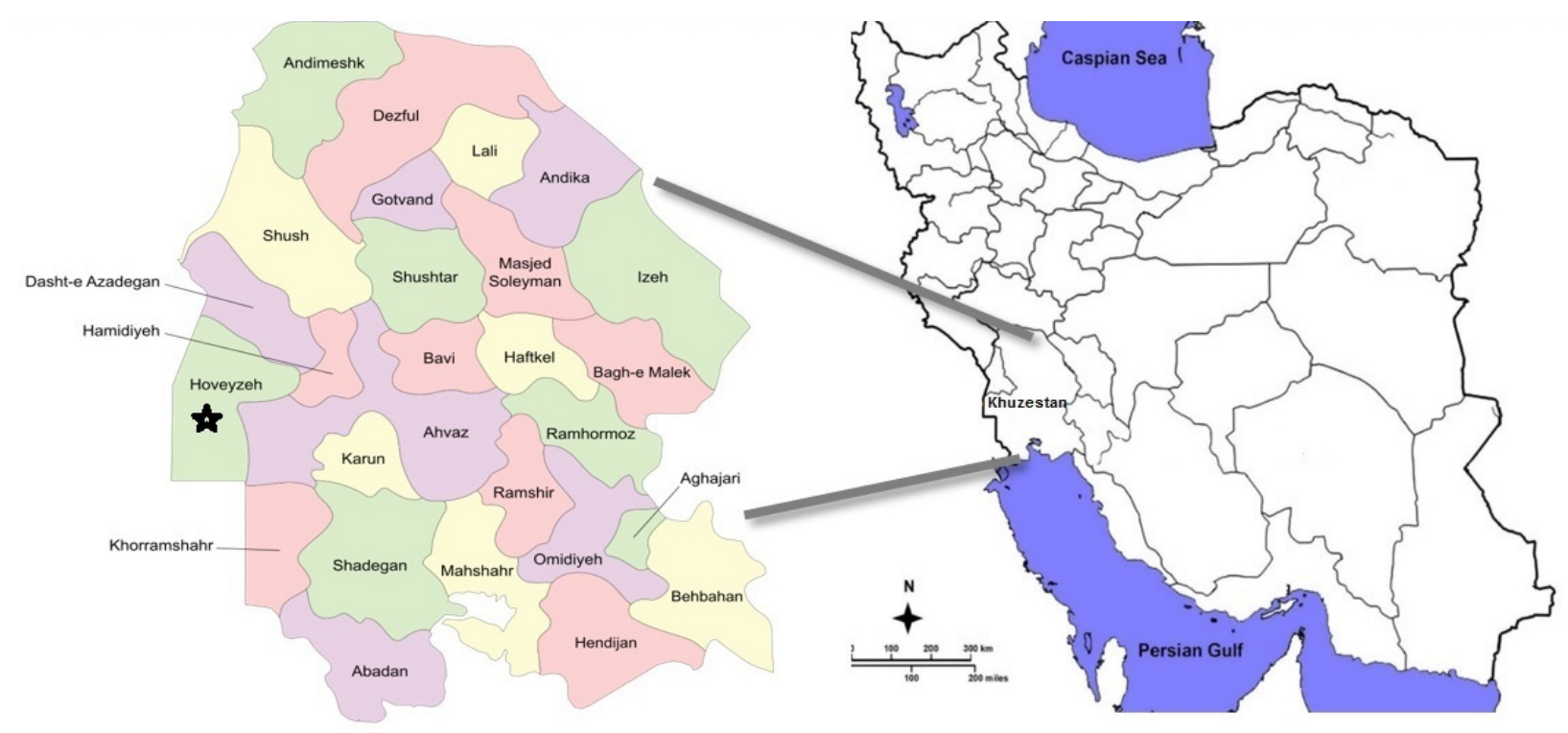

Fig. 1. Location of Hoveyzeh County 
study was partially provided by the Vice President of Research and Technology at the Ministry of Health and Medical Education and partially by Ahvaz Jundishapur University of Medical Sciences and a local charity organization.

\section{Who is in the cohort study?}

The HCS is a prospective population-based cohort study of 10009 adults (age 35-70 years) recruited from May 2016 to August 2018, designed to assess NCDs in southwest of Iran. The Hoveyzeh cohort center is one of the sites of the Prospective Epidemiological Research Studies in IrAN (the PERSIAN Cohort Study), including 180000 Iranian adults (7). Based on the 2016 door-to-door census, there were 7772 eligible individuals living in Hoveyzeh county: 4378 individuals in the 2 cities of Hoveyzeh and Rofayyeh (2187 males and 2191 females) and 3394 in 27 villages (1611 males, 1783 females). In addition, in 7 villages and 2 urban areas of Susangerd bordering Hoveyzeh, 4331 eligible people were invited (7).

Prior to the start of the project, several meetings were held with local authorities and trustees to familiarize them with the advantages and stages of the study to increase the participation rate of the local people. To introduce the PERSIAN Cohort Study, several lectures were held in public places such as mosques and cultural centers, and pamphlets were also distributed. Then, the census was conducted by local Red Crescent volunteers and sanitary inspectors. For this purpose, trained personnel recorded the required information of all eligible individuals. In addition, the geographical coordinates of each visited house were defined and recorded using the Garmin GPSMAP 78s. Invitations to the cohort site were given by trained inviters one week before the referral day. A phone call was made to remind the invitees the day before the visit. Participants were transported daily to the cohort site by a minibus.

Out of 12103 eligible individuals invited, 8792 were enrolled in the study for the first stage, 982 for the second stage, and 235 for the third stage; thus, 10009 individuals entered the study. The overall response proportion was $85.16 \%$ (Fig. 2). Responders and nonresponders are compared in terms of baseline variables in Table 1.

All the assessed socio demographic characteristics were significantly different between the responder and non responder groups. The distributions of gender and education level were obviously different in the 2 groups; the response proportion between women was nearly 1.5 times more than men. In addition, response proportion in the less educated individuals was considerably higher than in those more educated. However, it seems that the distributions of the other socio demographic characteristics were almost similar and the significant $P$ values may be ascribed to the large sample.

\section{How often have they been followed up?}

The follow-up will be conducted annually for at least 15 years after enrollment. This phase will be performed in 2 ways, including active follow-up (telephone call) and passive follow-up (self-reporting and report of death and disease by registration centers). The follow-up questionnaires will be_completed annually by the follow-up team involving trained midwives, nurses, and physicians, assessing the study's outcomes of interest: death, cause of death, and incidence of major NCD. In case of death, a verbal autopsy is performed to find the definite cause of death and in case of NCD incidence, all diagnostic and therapeutic documents are assessed and archived.

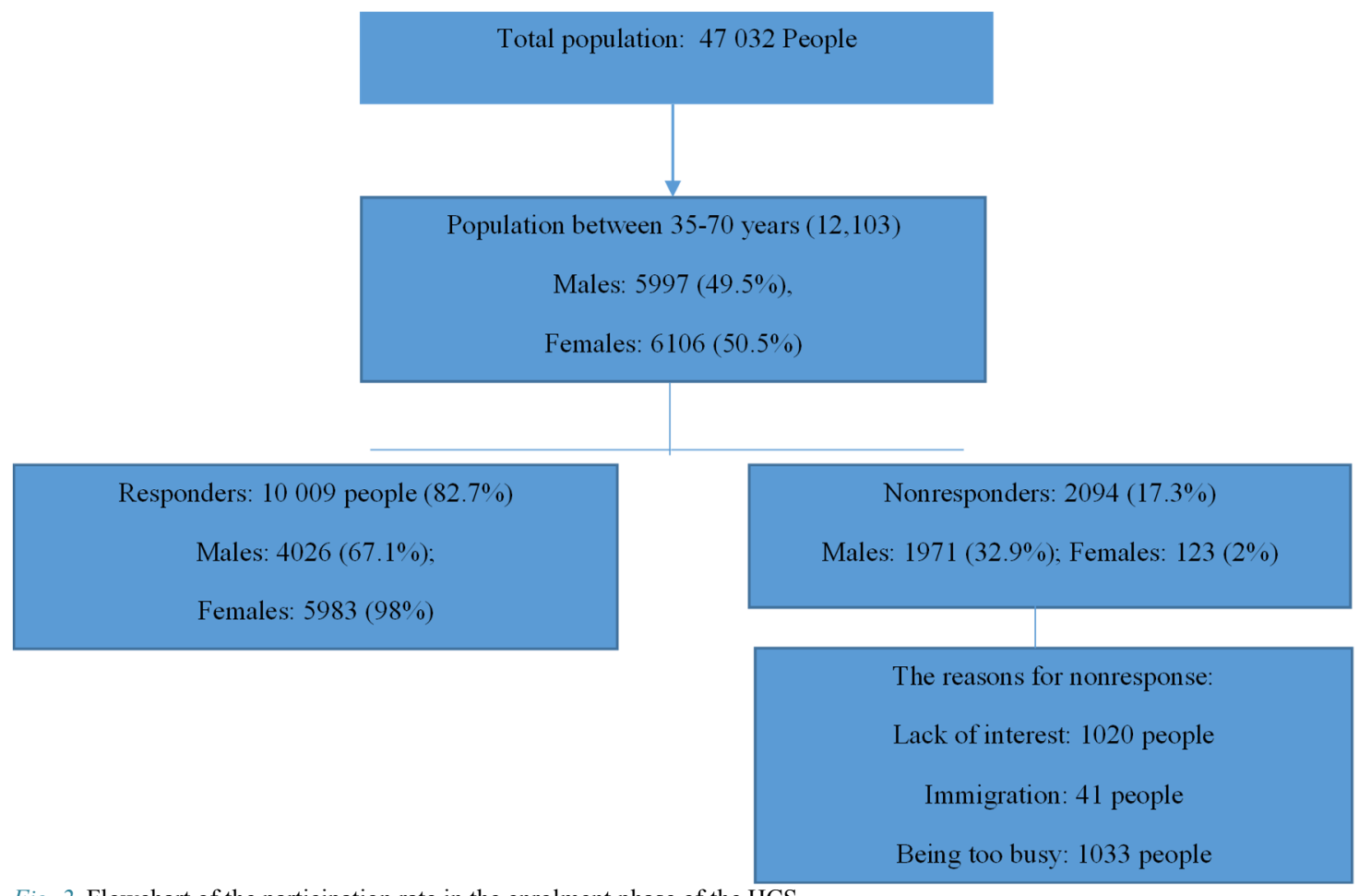

Fig. 2. Flowchart of the participation rate in the enrolment phase of the HCS 


\begin{tabular}{|c|c|c|c|}
\hline \multirow[t]{2}{*}{ Characteristic } & \multicolumn{2}{|c|}{ Response } & \multirow{2}{*}{$\begin{array}{c}\mathrm{P} \\
\text { (Chi-square) }\end{array}$} \\
\hline & Responders, number (\%) & Non-responders, number (\%) & \\
\hline \multicolumn{4}{|l|}{ Age group (year) } \\
\hline $35-39$ & 1912(19.1) & $462(22.1)$ & \\
\hline $40-44$ & $2025(20.2)$ & $477(23)$ & $<0.001$ \\
\hline $45-49$ & $1797(18)$ & $365(17.5)$ & \\
\hline $50-54$ & $1482(14.8)$ & $246(11.7)$ & \\
\hline $55-59$ & $1281(12.8)$ & $214(10.2)$ & \\
\hline $60-64$ & $798(8)$ & $195(9.3)$ & \\
\hline$\geq 65$ & $714(7.1)$ & $135(6.2)$ & \\
\hline \multicolumn{4}{|l|}{ Gender } \\
\hline Male & $4026(40.2)$ & 1971(94.1) & $<0.001$ \\
\hline Female & $5983(59.8)$ & $123(5.9)$ & \\
\hline \multicolumn{4}{|l|}{ Marital status } \\
\hline Married & $8760(87.5)$ & $1314(62.8)$ & $<0.001$ \\
\hline Widowed & $737(7.4)$ & $355(17)$ & \\
\hline Divorced & $169(1.7)$ & $243(11.6)$ & \\
\hline Single & $343(3.4)$ & $182(8.6)$ & \\
\hline \multicolumn{4}{|l|}{ Level of education } \\
\hline Illiterate & $6209(62)$ & $693(33.1)$ & \\
\hline Primary school & $1665(16.7)$ & $425(20.3)$ & $<0.001$ \\
\hline Secondary school & $673(6.7)$ & $337(16.1)$ & \\
\hline High school & $741(7.4)$ & $268(12.8)$ & \\
\hline Higher education & $721(7.2)$ & $371(17.7)$ & \\
\hline
\end{tabular}

\section{How often questionnaires will be conducted?}

Selected questionnaires will be completed for all the participants in the 5 th, 10th, and 15 th years of follow-up to assess changes in risk or protective factors.

\section{How often examinations will be conducted?}

Similar to the questionnaire completions, biological samples (blood, urine, hair and nail) will be collected in the 5th, 10 th, and 15th years of follow-up, and anthropometric indices will be measured. Whenever any case of myocardial infarction, stroke, or cancer occurs, an additional blood sample will be collected from the patient and will be stored in the Biobank.

\section{Response at baseline}

The overall response proportion at baseline was $85.16 \%$ (67.1\% for males and $98 \%$ for females).

\section{Loss to follow-up over time}

Given that the first-year of follow-up has not yet been completed, loss to follow-up has not yet been assessed.

How those lost to follow-up differ from those remaining in the cohort?

Given that the first-year of follow-up has not yet been completed, loss to follow-up has not yet been assessed.

\section{What has been measured?}

The HCS data were gathered through interviewer-administered questionnaires, physical examinations, and biological samples. This process took about 180 minutes per participant to complete. The measurement characteristics in HCS are presented in Table 2.

Enrolment phase: This phase consists of 8 steps shown in Figure 3.

\section{STEP 1: Registration}

On the day of the referral, participants were welcomed and once more informed about the importance of the study and all procedures involved. Written informed consent was obtained and individuals were enrolled using their national ID cards. Each participant was assigned an 11-digit code (PCID). These codes were used to label all biological samples and documents.

\section{STEP 2: Biological sampling}

Individuals attending the study had been fasting for about 10 to 12 hours on the day of enrollment. Immediately after enrollment, blood, urine, hair, and nail samples were taken by trained laboratory staff. First, 25 milliliters of blood, including one clot tube $(6 \mathrm{ml})$ and two k2-EDTA tube $(9 \mathrm{~mL})$ (KANG JIAN, China) by Vacutainers (Greiner Bio-One International $\mathrm{GmbH}$, Kremsmunster, Austria) and urine (15$25 \mathrm{~mL}$ ) were collected from each participant. Hair (300 to 500 strands, length $1-3 \mathrm{~cm}$, from the back of the head) and nail (from all fingers) samples were also taken from all participants. These samples were stored as explained in Table 2.

In addition to sample storage, blood samples were centrifuged (Sigma, Germany) at $3000 \mathrm{rpm}$ for 10 minutes to separate serum. Then, the required serum levels were measured by BT 1500 autoanalyser (Biotecnica Instruments, Italy). A complete blood count ( $\mathrm{CBC}$ ) was done by the hematology autoanalyser (Nihon Kohden 6510-k, Japan). Urine tests (urine $\mathrm{pH}$, specific gravity) and analysis were also performed. Hair and nail samples were separately stored in a piece of foil, which was then placed inside a ziplock bag containing silica gels for moisture control. The bags are kept in a cool and dry location at the HCS Biobank.

STEP 3: Anthropometric measurements

Since the error or bias in measurements is minimal in the 


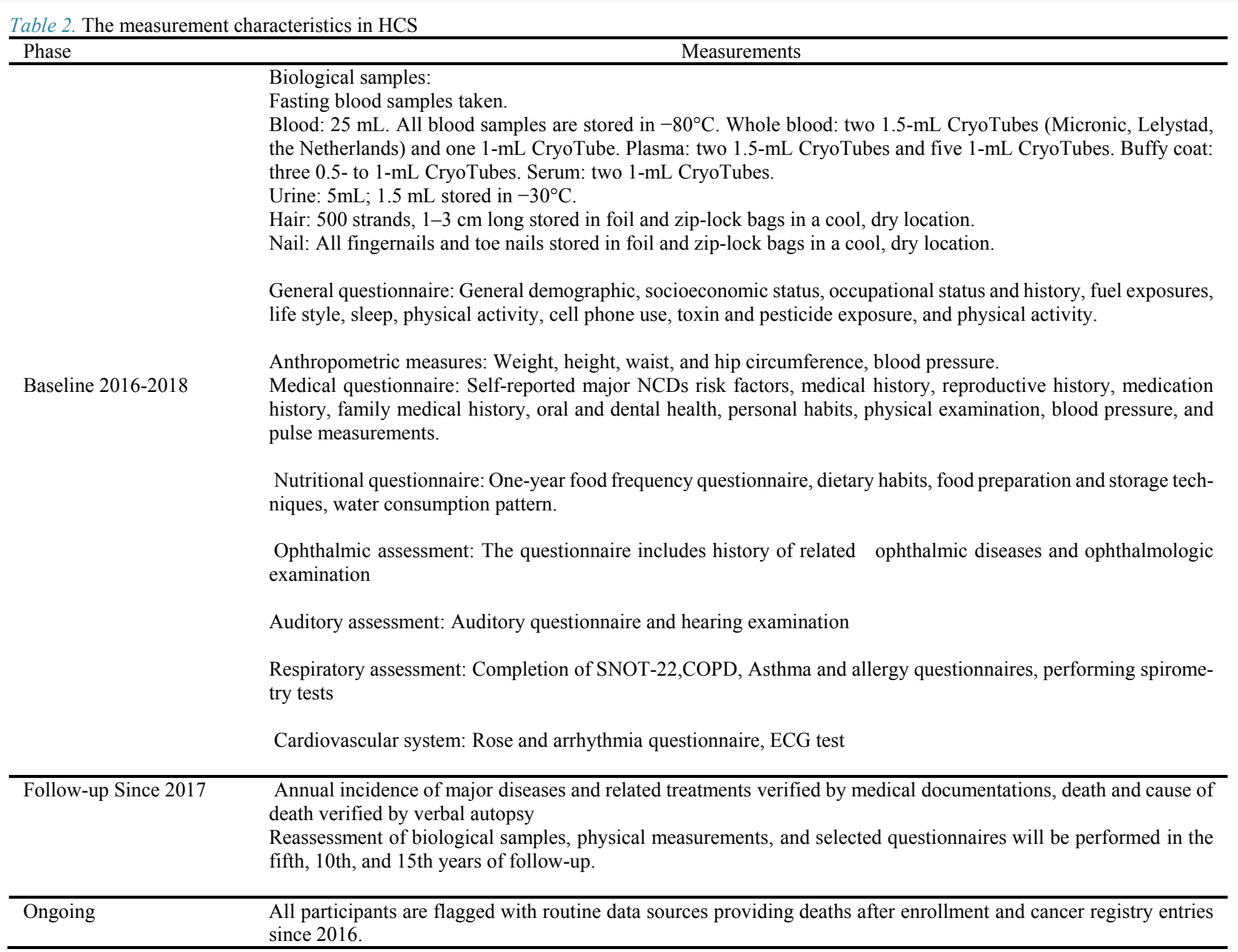

morning, anthropometric data were measured after biological sampling, while the participants were still in a fasting

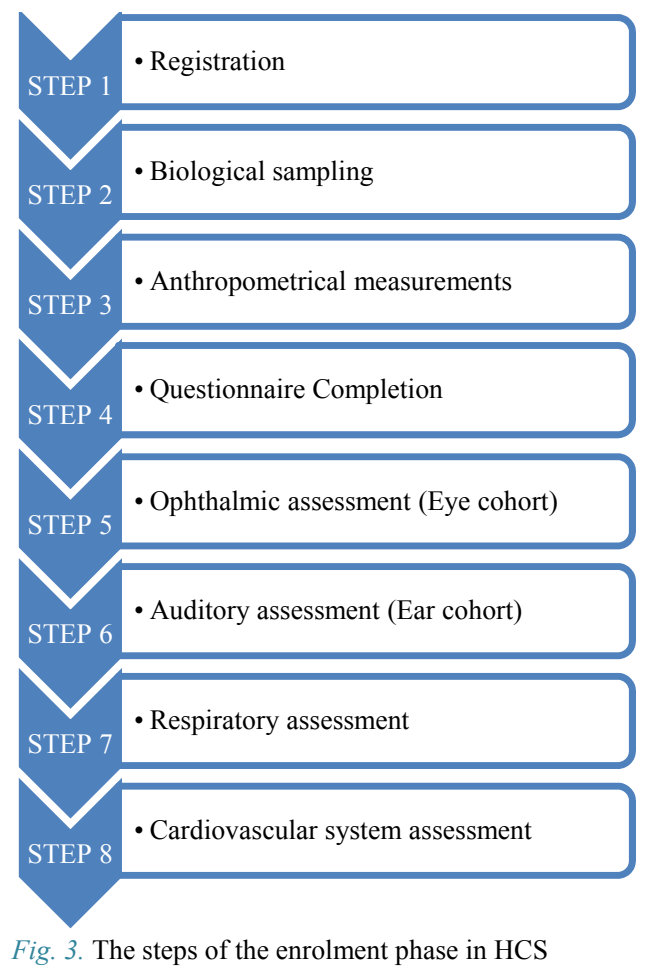

state. Height (in $\mathrm{cm}$ ) was measured using a stadiometer (Seca 206), weight (in $\mathrm{kg}$ ) was measured using a standing scale (Seca 755), and waist, hip, and wrist circumferences (in $\mathrm{cm}$ ) were measured using Seca locked tape meters. Then, the participants were served breakfast prior to the questionnaire interviews.

\section{STEP 4: Filling in the questionnaires}

The PERSIAN cohort questionnaires include General Nutrition and Medical sections. All questionnaires were completed by interviewers. Categories of topics in the PERSIAN cohort questionnaires are shown in Table 3. As part of a physical examination, blood pressure was measured by Riester sphygmomanometers, twice (10 min interval) on each arm following standard guidelines. Pulse rate was also measured twice for 60 seconds.

\section{STEP 5: Ophthalmology and ophthalmic examinations (Eye cohort)}

The PERSIAN Eye Cohort is being conducted in only 6 of the 19 PERSIAN Cohort sites, including Hoveyzeh. An ophthalmologist, an optometrist, and an expert were present at the HCS site, where a questionnaire covering several items on the history of diabetes, a family history of major and common ocular diseases, eye surgery, last ophthalmologic examination time, and possible vision complaints was completed. Then, ophthalmic examinations consisting of 


\begin{tabular}{lc} 
Table 3. Categories of topics in the PERSIAN Cohort Questionnaire, Iran, 2016-2018 & Number of questions \\
\hline Questionnaire & 42 \\
\hline General demographic factors & 27 \\
Socioeconomic status (current) & 7 \\
Occupational status and history & 9 \\
Fuel exposures (past and current) & 13 \\
Lifestyle (past and current) & 11 \\
Sleep and circadian rhythm (in the past year) & 28 \\
Physical activity (in the past year) & 4 \\
Cell phone use (past and current) & 10 \\
Toxin and pesticide exposure (in the past year) & 23 \\
Medical history & 33 \\
Reproductive history (women) & 81 \\
Medication history & 27 \\
Family medical history & 9 \\
Oral and dental health (past and current) & 4 \\
Personal habits (smoking, alcohol and drug use) (past and current) & 11 \\
Blood pressure and pulse measurements & 8 \\
Physical examination & 124 \\
Nutrition Food frequency questionnaire (130 items in the past year) & 29 \\
Dietary habits (current) food preparation and storage techniques (current) & 2 \\
Water use & \\
\hline
\end{tabular}

lensometry of current glasses (if applicable), testing near/far visual acuity, determining objective and subjective refraction, eye motility and cycloplegic refraction were performed.

\section{STEP 6: Assessment of the auditory system (Ear cohort)}

Hoveizeh "Ear Cohort" is the only auditory sub cohort study in the PERSIAN Cohort Study. After filling out a detailed case-history (35 questions), all subjects were undergoing acoustic impedance and pure tone audiometry. The acoustic impedance test is a beneficial procedure for the assessment of conductive components of the auditory system (e.g., tympanic membrane) and was conducted using a calibrated tympanometer (AT235 model, Intracoustics, Denmark). The conventional pure-tone audiometry (PTA) and extra-high frequency PTA (EHF-PTA) were used to measure hearing thresholds at $250-8000 \mathrm{~Hz}$ and $10000-16000$ $\mathrm{Hz}$ frequency range, respectively. A calibrated diagnostic audiometer (AC40 model, Intracoustics, Denmark) coupled with a standard (Telephonic TDH 39, Supra-aural) and extra high-frequency (Sennheiser HDA-200, Circum-aural) headphones were utilized for hearing threshold evaluations. All audiological tests were conducted in a sound-attenuated booth.

\section{STEP 7: Assessment of respiratory system}

To further assess the impact of environmental and dust storms effects on respiratory system additional testing was performed at HCS. Pulmonary function was assessed in all participants using a spirometer (model Spiro Scout; GANSHORN) For Estimating the prevalence of respiratory diseasese such as asthma, COPD and chronic allergic rhinosinositis in adults, and long-term trend of asthma and wheezing, and studying the potential risk factors for the incidence and persistence of wheezing and other respiratory disorders, for which the SNOT-22, COPD, Asthma and Allergy questionnaires were completed (9). Two trained technicians completed the questionnaires and performed the spirometry tests.

\section{STEP 8: Assessment of cardiovascular system}

ECGs were taken by 2 trained staff using a DG7000 12channel electrocardiograph (D.G. Dena Co.). The ECGs were recorded in $16 \mathrm{~s}$ and $32 \mathrm{~s}$ phases. In addition, the WHO Rose 5-item questionnaire (10) and Heartbeat assessment questionnaire were completed for each participant. ECG results were sent to a cardiologist for cardiac problem assessment. At the completion of all enrolment steps, participants received a cohort identification card for future use.

What have been found? Key findings and publications

In the enrollment phase, 10009 people were enrolled in the study from May 23, 2016 to August 28, 2018. Table 4 displays the self-reported prevalence of health conditions among the participants.

Diabetes is defined as having a fasting blood sugar $126 \mathrm{mg} / \mathrm{dL}$ and greater or using glucose lowering medications or self-reported diagnosis of diabetes. Hypertension is defined as having a systolic blood pressure above $140 \mathrm{mmHg}$ or diastolic blood pressure above $90 \mathrm{mmHg}$ or using blood pressure lowering medications or self-reported diagnosis of hypertension. Criteria of metabolic syndrome consist of abdominal obesity (waist circumstance equal and more than 102 in men and equal and more than 88 in women), serum triglycerides (equal and more than $150 \mathrm{mg} / \mathrm{dL}$ ), or using hypertriglyceridemia medications, serum high density lipoprotein (HDL) cholesterol (equal and more than $40 \mathrm{mg} / \mathrm{dL}$ in men and equal and more than 50 in women), or using drug treatment for low HDL cholesterol, blood pressure $\geq 130 / 85 \mathrm{mmHg}$, or using hypertension drugs, fasting plasma glucose $(\mathrm{FPG}) \geq 100 \mathrm{mg} / \mathrm{dL}$, or using hyperglycemia drugs.

The presence of any 3 of the 5 above criteria constitutes a diagnosis of metabolic syndrome (11).

Table 5 demonstrates the prevalence of NCDs risk factors among participants. An active smoker is defined as a person who has smoked more than 100 cigarettes in his or her lifetime and passive smokers are those who inhale cigarette smoke indirectly; for example, as a second-hand smoker. To determine drug and alcohol abuse among participants, 


\begin{tabular}{|c|c|c|c|}
\hline \multirow[t]{2}{*}{ Conditions } & \multicolumn{3}{|c|}{$\mathrm{N}(\%)$} \\
\hline & Male (prevalence) & Female (prevalence) & Total \\
\hline Diabetes & $883(21.9)$ & $1343(22.4)$ & $2226(22.2)$ \\
\hline High blood pressure & $943(23.4)$ & $1700(28.4)$ & $2643(26.4)$ \\
\hline Metabolic Syndrome & $1180(29.3)$ & $2733(45.7)$ & $3913(39.1)$ \\
\hline Cardiac Ischemic & $447(11.1)$ & $913(15.3)$ & $1360(13.6)$ \\
\hline MI History & $88(2.2)$ & $97(1.6)$ & $185(1.85)$ \\
\hline Stroke History & $70(1.7)$ & $89(1.5)$ & $159(1.6)$ \\
\hline Renal Failure & $53(1.3)$ & $70(1.2)$ & $123(1.2)$ \\
\hline Fatty Liver & $222(5.5)$ & $453(7.6)$ & $675(6.7)$ \\
\hline Chronic Lung Disease & $211(5.2)$ & $374(6.3)$ & $585(5.8)$ \\
\hline Thyroid disorders & $80(2)$ & $460(7.7)$ & $540(5.4)$ \\
\hline Kidney Stone & $976(24.2)$ & $901(15.1)$ & $1877(18.8)$ \\
\hline Gallstone & $55(1.4)$ & $242(4)$ & $297(3.0)$ \\
\hline Rheumatic Disease & $62(1.53)$ & $375(6.3)$ & $437(4.4)$ \\
\hline Chronic Headaches & $170(4.2)$ & $580(9.7)$ & $750(7.5)$ \\
\hline Gestational Hypertension History & $0(0.00)$ & $336(5.6)$ & $336(5.6)$ \\
\hline Gestational Diabetes History & $0(0.00)$ & $221(3.7)$ & $221(3.7)$ \\
\hline
\end{tabular}

Table 5. Baseline prevalence of common NCD risk factors among HCS participants

\begin{tabular}{|c|c|c|c|}
\hline \multirow[t]{2}{*}{ Risk Factors } & \multicolumn{3}{|c|}{$\mathrm{N}(\%)$} \\
\hline & Male (prevalence) & Female (prevalence) & Total \\
\hline Smoker & $1635(40.6)$ & $454(7.6)$ & $2089(20.9)$ \\
\hline Alcoholic & $191(4.7)$ & $6(0.1)$ & $197(2.0)$ \\
\hline Drug abuse & $203(5.0)$ & $4(0.07)$ & $207(2.1)$ \\
\hline \multicolumn{4}{|l|}{ Wealth } \\
\hline Poorest & $647(16)$ & $1353(22.6)$ & $2000(20)$ \\
\hline Poor & $729(18)$ & $1304(21.8)$ & $2033(22.3)$ \\
\hline Moderate & $821(20.4)$ & $1161(19.4)$ & $1982(19.8)$ \\
\hline Rich & $880(22)$ & $1143(19.1)$ & $2023(20.2)$ \\
\hline Richest & $949(23.6)$ & $1022(17.1)$ & $1971(19.7)$ \\
\hline \multicolumn{4}{|l|}{ BMI } \\
\hline Under weight & $70(1.7)$ & $79(1.2)$ & $149(1.5)$ \\
\hline Normal & $1146(28.5)$ & $1097(18.3)$ & $2243(22.4)$ \\
\hline Over weight & $1706(42.4)$ & $2006(33.5)$ & $3712(37.1)$ \\
\hline Obese & $1104(27.4)$ & $2801(47)$ & $3905(39)$ \\
\hline \multicolumn{4}{|c|}{ Waist to hip ratio (WHR) } \\
\hline Normal $^{*}$ & $584(14.5)$ & $396(6.6)$ & $980(9.8)$ \\
\hline Abnormal & $3442(85.5)$ & $5587(93.4)$ & $9029(90.2)$ \\
\hline \multicolumn{4}{|l|}{ Physical activity } \\
\hline Quartile 1 & $1255(31.2)$ & $1248(20.9)$ & $2503(25.02)$ \\
\hline Quartile 2 & $734(18.2)$ & $1771(29.6)$ & $2505(25.03)$ \\
\hline Quartile 3 & $650(16.1)$ & $1858(31)$ & $2508(25.05)$ \\
\hline Quartile 4 & $1387(34.5)$ & $1106(18.5)$ & 2493 (24.9) \\
\hline \multicolumn{4}{|l|}{ FBS } \\
\hline Less than 100 & $2458(61.1)$ & $3529(59)$ & $5987(59.8)$ \\
\hline $100-125$ & $842(20.9)$ & $1469(24.5)$ & $2311(23.1)$ \\
\hline$\geq 126$ & $726(18)$ & $985(16.5)$ & $1711(17.1)$ \\
\hline \multicolumn{4}{|l|}{ Triglyceride } \\
\hline Less than 150 & 1948 (48.4) & 3663 (61.2) & $5611(56.1)$ \\
\hline 150 and more & $2078(51.6)$ & $2320(38.8)$ & $4398(43.9)$ \\
\hline \multicolumn{4}{|l|}{ Cholesterol } \\
\hline Less than 200 & $2628(65.3)$ & $3751(62.7)$ & $6379(63.7)$ \\
\hline 200 and more & $1398(34.7)$ & $2232(37.3)$ & $3630(36.3)$ \\
\hline \multicolumn{4}{|c|}{ Cholesterol HDL } \\
\hline Normal $^{* *}$ & $2917(72.5)$ & $3459(57.8)$ & $6376(63.7)$ \\
\hline Abnormal & $1109(27.5)$ & $2524(42.2)$ & $3633(36.3)$ \\
\hline
\end{tabular}

${ }^{*}$ A healthy WHR for women is 0.85 or less and a healthy WHR for men is 0.90 or less

${ }_{* *}^{*}$ Normal HDL is defined as 50 and more for female and 40 and more for male

we asked whether they have ever used them in their lifetime. The amount of consumption and its type have also been asked.

BMI is defined as a person's weight in kilograms divided by the square of the person's height in meters $(\mathrm{kg} / \mathrm{m} 2)$. A BMI of below 18.5 is underweight, while the healthy range is 18.5 to $24.9,25.0-29.9$ is overweight, and more than 30 is considered as obese. The average of body mass index
(BMI) in males was $27.5 \pm 4.6$ and it was $29.7 \pm 5.54$ in females. Another measurement of obesity is waist-to-hip ratio (WHR), which in turn is a possible indicator of other more serious health conditions. The average of WHR was $0.96 \pm 0.06$ in males and $0.95 \pm 0.07$ in females.

Wealth index as an individual level of socioeconomic status was calculated by means of information on households' possession, including freezer, TV, motorbike, cell phone, 
car, and vacuum cleaner, access to internet, washing machine, computer and household utilities including house ownership, number of rooms per capita, were entered to into a principal component analysis (PCA) and finally the score of wealth index was converted to 5-ordered categories from poorest to richest (12).

To measure the physical activity of participants, the metabolic equivalent of task (MET Index) was calculated to express the intensity of physical activities. MET is the ratio of a person's working metabolic rate relative to their resting metabolic rate. One MET is defined as the energy cost of sitting quietly and is equivalent to a caloric consumption of $1 \mathrm{kcal} / \mathrm{kg} / \mathrm{hour}$. The daily physical activity questionnaire used to measure MET for all participants' activities during 24 hours a day and quartiles were calculated for all participants.

\section{Quality control in the HCS}

Quality control teams have been established at the central (PERSIAN Cohort), university and local levels to ensure good quality data collection. In all stages, including census, invitation, enrolment, biological sampling, interviewing, examinations, data entry as well as follow-up processes, are continuously being monitored and supervised directly by the quality control teams. In addition, at the end of the interview process, some of the participants were randomly asked to go to the field observer room and recomplete the questionnaire (at least 2 questions from each domain). The field observer monitored the accuracy of the data recorded in the online system and reviewed the participants' responses. Cleaning of the collected data is continually done by both university and central quality control teams. In addition, an external observer independently monitored all stages of data collection and then reported the results to the local and central teams each month.

\section{What are the main strengths and weaknesses?}

Participation rate was high in the enrolment phase of the study. Ethnicity and life style of Iranian Arabs is almost the same as people of neighbor countries like Iraq and Kuwait, so the findings from the HCS can be generalized to a wide geographical area covering millions of people. In addition, immigrant acceptance or immigration rates are very low in this city. Wide ranges of variables are considered in the study, which can be used to design numerous research projects in the future, assessing the relationship between several conditions and medical, demographic, genetic, and laboratory variables. The quality control of this study was precisely performed at national, university, and field levels. Compared to the other PERSIAN Cohort sites, additional assessments on cardiovascular and respiratory diseases as well as hearing and visual disorders have been performed in $\mathrm{HCS}$.

Despite several strengths, the present study has some limitations. This cohort will not be able to assess rare outcomes at the local level because of its limited sample size. The studied sample is restricted to the 35-70-year age group; therefore, the results cannot be generalized to younger or older age groups.

\section{Can I get the data? Where can I find out more?}

We are open to accept offers for collaboration. Therefore, specific proposals from national and international researchers are welcomed. The submitted proposals will be discussed within the steering committee of the HCS. The researchers will have access to the data after the approval of their proposals in this committee and the ethics committee of Ahvaz Jundishapur University of Medical Sciences. Detailed information about the study and submission process is available at cohort.ajums.ac.ir. We invite all collaborators interested in using the data to contact the chief researchers, Nader Saki (ahvaz.ent@gmail.com).

\section{Acknowledgement}

The Iranian Ministry of Health and Medical Education has contributed to the funding used in the PERSIAN Cohort through Grant no. 700/534. This project was funded and approved by Ahvaz Jundishapur University of Medical Sciences (Ethical code: IR.AJUMS.REC.1396.149).

\section{Conflict of Interests}

The authors declare that they have no competing interests.

\section{References}

1. Fact sheet Noncommunicable diseases. 1 June 2018.

2. Global, regional, and national comparative risk assessment of 84 behavioural, environmental and occupational, and metabolic risks or clusters of risks for 195 countries and territories, 1990-2017: a systematic analysis for the Global Burden of Disease Study 2017. Lancet (London, England). 2018;392(10159):1923-94.

3. IRAN (ISLAMIC REPUBLIC OF) [Available from: https://www.who.int/nmh/countries/irn_en.pdf?ua=1IRAN

4. Global, regional, and national incidence, prevalence, and years lived with disability for 354 diseases and injuries for 195 countries and territories, 1990-2017: a systematic analysis for the Global Burden of Disease Study 2017. Lancet (London, England). 2018;392(10159):1789-858.

5. Vandenberghe D, Albrecht J. The financial burden of noncommunicable diseases in the European Union: a systematic review. Eur J Public Health. 2019.

6. Rao SS, Singh RB, Takahashi T, Juneja LR, Fedacko J, Shewale AR. Chapter 4 - Economic Burden of Noncommunicable Diseases and Economic Cost of Functional Foods for Prevention. In: Singh RB, Watson RR, Takahashi T, editors. The Role of Functional Food Security in Global Health: Academic Press; 2019. p. 57-68.

7. Poustchi H, Eghtesad S, Kamangar F, Etemadi A, Keshtkar AA, Hekmatdoost A, et al. Prospective Epidemiological Research Studies in Iran (the PERSIAN Cohort Study): Rationale, Objectives, and Design. Am J Epidemiol. 2018;187(4):647-55.

8. Maleki H, Sorooshian A, Goudarzi G, Nikfal A, Baneshi MM. Temporal profile of PM10 and associated health effects in one of the most polluted cities of the world (Ahvaz, Iran) between 2009 and 2014. Aeolian Res. 2016 Sep;22:135-140.

9. van den Berge M, Ten Hacken NHT, Cohen J, Douma WR, Postma DS. Small airway disease in asthma and COPD: clinical implications. Chest. 2011;139(2):412-23.

10. Najafi-Ghezeljeh T, Kassaye Tessama M, Yadavar-Nikravesh M, Ekman I, Emami A. The Iranian version of Angina Pectoris characteristics questionnaire: reliability assessment. J Clin Nurs. 2009;18(5):694-9.

11. Metabolic syndrome (insulin resistance syndrome or syndrome $\mathrm{X}$ ) [Internet]. Jul 30, 2020. Available from: https://www.uptodate.com/contents/metabolic-syndrome-insulinresistance-syndrome-or-syndrome-x.

12. Smits J, Steendijk R. The International Wealth Index (IWI). Soc Indic Res. 2015;122(1):65-85. 